\title{
Endothelial CCR6 expression due to FLI1 deficiency contributes to vasculopathy associated with systemic sclerosis
}

\author{
Tetsuya Ikawa, Takuya Miyagawa, Yuki Fukui, Satoshi Toyama, Jun Omatsu, Kentaro Awaji, Yuta Norimatsu, \\ Yusuke Watanabe, Ayumi Yoshizaki, Shinichi Sato and Yoshihide Asano* (1)
}

\begin{abstract}
Background: We have recently demonstrated that serum CCL20 levels positively correlate with mean pulmonary arterial pressure in patients with systemic sclerosis (SSC). Considering a proangiogenic effect of CCL20 on endothelial cells via CCR6, the CCL20/CCR6 axis may contribute to the development of SSC vasculopathy. Therefore, we explored this hypothesis using clinical samples, cultured cells, and murine SSC models.

Methods: The expression levels of CCL20 and CCR6 in the skin, mRNA levels of target genes, and the binding of transcription factor FLI1 to the target gene promoter were evaluated by immunostaining, quantitative reverse transcription PCR, and chromatin immunoprecipitation, respectively. Vascular permeability was evaluated by Evans blue dye injection in bleomycin-treated mice. Angiogenic activity of endothelial cells was assessed by in vitro angiogenesis assay.

Results: CCL20 expression was significantly elevated in dermal fibroblasts of patients with early diffuse cutaneous SSc, while CCR6 was significantly up-regulated in dermal small vessels of SSC patients irrespective of disease subtypes and disease duration. In human dermal microvascular endothelial cells, FLI1 siRNA induced the expression of CCR6, but not CCL20, and FLI1 bound to the CCR6 promoter. Importantly, vascular permeability, a representative SSc-like vascular feature of bleomycin-treated mice, was attenuated by Ccr6 siRNA treatment, and CCR6 siRNA suppressed the angiogenic activity of human dermal microvascular endothelial cells assayed by in vitro tube formation.
\end{abstract}

Conclusions: The increased expression of endothelial CCR6 due to FLI1 deficiency may contribute to the development of SSc vasculopathy.

Keywords: Systemic sclerosis, CCL20, CCR6, FLI1, Endothelial cells

\section{Background}

Systemic sclerosis (SSc) is a multisystem autoimmune disease representing vasculopathy and tissue fibrosis of the skin and various internal organs [1]. Recent clinical studies have demonstrated the efficacy of several drugs for tissue fibrosis and vasculopathy associated with SSc.

*Correspondence: yasano-tky@umin.ac.jp Department of Dermatology, University of Tokyo Graduate School of Medicine, Tokyo, Japan
For instance, tocilizumab and bosentan prevent the decrease in the percentage of vital capacity and the onset of new digital ulcers, respectively [2, 3]. Also, several new therapeutic candidates are now under the clinical trials [4], but the identification of new therapeutic targets is quite important to further facilitate the development of new therapies against SSc.

Chemokines have attracted much attention as a potential therapeutic target family of molecules in the field of SSc based on the results of clinical studies and animal original author(s) and the source, provide a link to the Creative Commons licence, and indicate if changes were made. The images or other third party material in this article are included in the article's Creative Commons licence, unless indicated otherwise in a credit line to the material. If material is not included in the article's Creative Commons licence and your intended use is not permitted by statutory regulation or exceeds the permitted use, you will need to obtain permission directly from the copyright holder. To view a copy of this licence, visit http://creativecommons.org/licenses/by/4.0/. The Creative Commons Public Domain Dedication waiver (http://creativeco mmons.org/publicdomain/zero/1.0/) applies to the data made available in this article, unless otherwise stated in a credit line to the data. 
models [5]. With respect to CCL20, we have recently found that serum CCL20 levels correlate with mean pulmonary arterial pressure (mPAP) in SSc patients [6], suggesting that the CCL20/CCR6 axis underlies the developmental mechanism of SSc vasculopathy. According to previous studies, the CCL20/CCR6 axis promotes the chemotaxis of immature dendritic cells, $\mathrm{T}$ helper (Th) 17 cells, regulatory $\mathrm{T}$ cells, and B cells under both homeostatic and inflammatory conditions [7], contributing to the maintenance of homeostatic immune balance and the development of pathologic inflammation, such as psoriasis [8, 9], atopic dermatitis [9], inflammatory bowel disease [10], systemic lupus erythematosus [11], dermatomyositis/polymyositis [12], and SSc [13]. On the other hand, an accumulating body of evidence indicates that the CCL20/CCR6 axis regulates endothelial behaviors related to tumor angiogenesis. For instance, Hippe et al. revealed the following findings: (i) CCL20 expression levels in tumors correlate with advanced tumor stage, increased lymph node metastasis, and decreased survival; (ii) microvascular endothelial cells abundantly express CCR6; (iii) CCR6 signaling in endothelial cells induces angiogenesis; and (iv) tumor growth and tumor-associated vascularization are decreased in CCR6-deficient mice due to its deficiency in stromal cells, but not within the immune system [14]. Thus, the CCL20/CCR6 axis is involved in the development of inflammatory diseases and tumor angiogenesis, but its role remains unknown in vascular disorders, including SSc vasculopathy.

Friend leukemia virus integration 1 (FLI1) is a member of the ETS transcription factor family, the expression of which is broadly suppressed in various cell types in SSc lesional and non-lesional skin [15, 16]. Since FLI1 expression is genetically and epigenetically suppressed in SSc patients [17, 18], FLI1 deficiency likely serves as a predisposing factor in SSc development. Supporting this notion, FLI1 deficiency induces SSc-like phenotypes in dermal fibroblasts, endothelial cells, macrophages, keratinocytes, and dermal dendritic cells [16, 19-25]. Therefore, the molecular analysis based on FLI1 deficiency provides us with a useful clue to know the significance of target molecules in the development of SSc. Indeed, this research strategy has been already applied to the study of chemokines in SSc. For instance, FLI1 deficiency induces CXCL5 upregulation and CXCL6 downregulation in endothelial cells [26, 27]. In addition, CXCL13 expression is enhanced by FLI1 deficiency in macrophages [24]. Importantly, the analyses on serum levels of these chemokines suggest their contribution to the development of tissue fibrosis, vasculopathy, and/ or immune activation in SSc. On the other hand, FLI1 deficiency is also implicated in the development of pulmonary arterial hypertension $(\mathrm{PAH})$ by modulating the expression of angiogenesis-related genes [28]. Taken together with our recent data regarding the association of the CCL20/CCR6 axis with SSc-PAH [6], we hypothesized that FLI1 deficiency regulates the CCL20/CCR6 axis in the context of SSc vasculopathy.

Based on these backgrounds, we investigated the potential role of CCL20/CCR6 in SSc vasculopathy and the contribution of FLI1 deficiency to this process by a series of experiments with clinical samples, cultured endothelial cells, and animal models.

\section{Methods}

\section{Immunohistochemistry}

Immunohistochemistry was performed on formalinfixed, paraffin-embedded skin sections with Vectastain Elite $\mathrm{ABC}$ kit (Vector Laboratories, Burlingame, CA, USA) and antibodies against CCL20 (Thermo Fisher Scientific, Waltham, MA, USA), CCR6 (Abcam, Cambridge, UK), $\alpha$-smooth muscle actin ( $\alpha$-SMA) (Abcam), NG2 (Abcam), vascular endothelial (VE)-cadherin (Thermo Fisher Scientific), and cleaved caspase 3 (Cell Signaling Technology, Danvers, MA, USA). Antigen retrieval was performed using Dako Target Retrieval Solution $\mathrm{pH} 9$ (Dako North America, Inc., Carpinteria, CA, USA). Skin samples were obtained from forearms of 6 diffuse cutaneous SSc (dcSSc) patients, 6 limited cutaneous SSc (lcSSc) patients, and 5 healthy controls. Patients' demographic features are shown in Table 1. All patients with dcSSc were the early subtype (disease duration of $<2$ years). Horseradish peroxidase activity was detected by 3 , 3'-diaminobenzidine. Counterstaining was conducted with methyl green. Manual scoring of staining intensity was graded as follows: - , no staining; + , slight staining; ++ , moderate staining; +++ , strong staining. Capillaries and venules were determined based on their diameter. Blood vessels with diameter almost equal to or less than that of erythrocyte were classified as capillaries, and the others were classified as venules [29]. The manual scoring of staining intensity and the determination of blood vessel type were performed by two independent dermatologists in a blinded manner (T. Ikawa and Y. Asano).

\section{Gene silencing of $F L I 1$}

Human dermal microvascular endothelial cells (HDMECs) (Lonza, Walkersville, MD, USA) were cultured on collagen-coated tissue culture plates in Endothelial Basal Medium-2 supplemented with the Endothelial Cell Growth Medium-2 Bullet Kit (Lonza). Shortly after seeded, HDMECs were transfected with FLI1 siRNA or non-silencing scrambled RNA (SCR) $(10 \mathrm{nM}$, both purchased from Santa Cruz Biotechnology, Santa Cruz, CA, USA) mixed with HiPerFect Transfection Reagent (Qiagen, Valencia, CA, USA) for $48 \mathrm{~h}$. Some cells were 


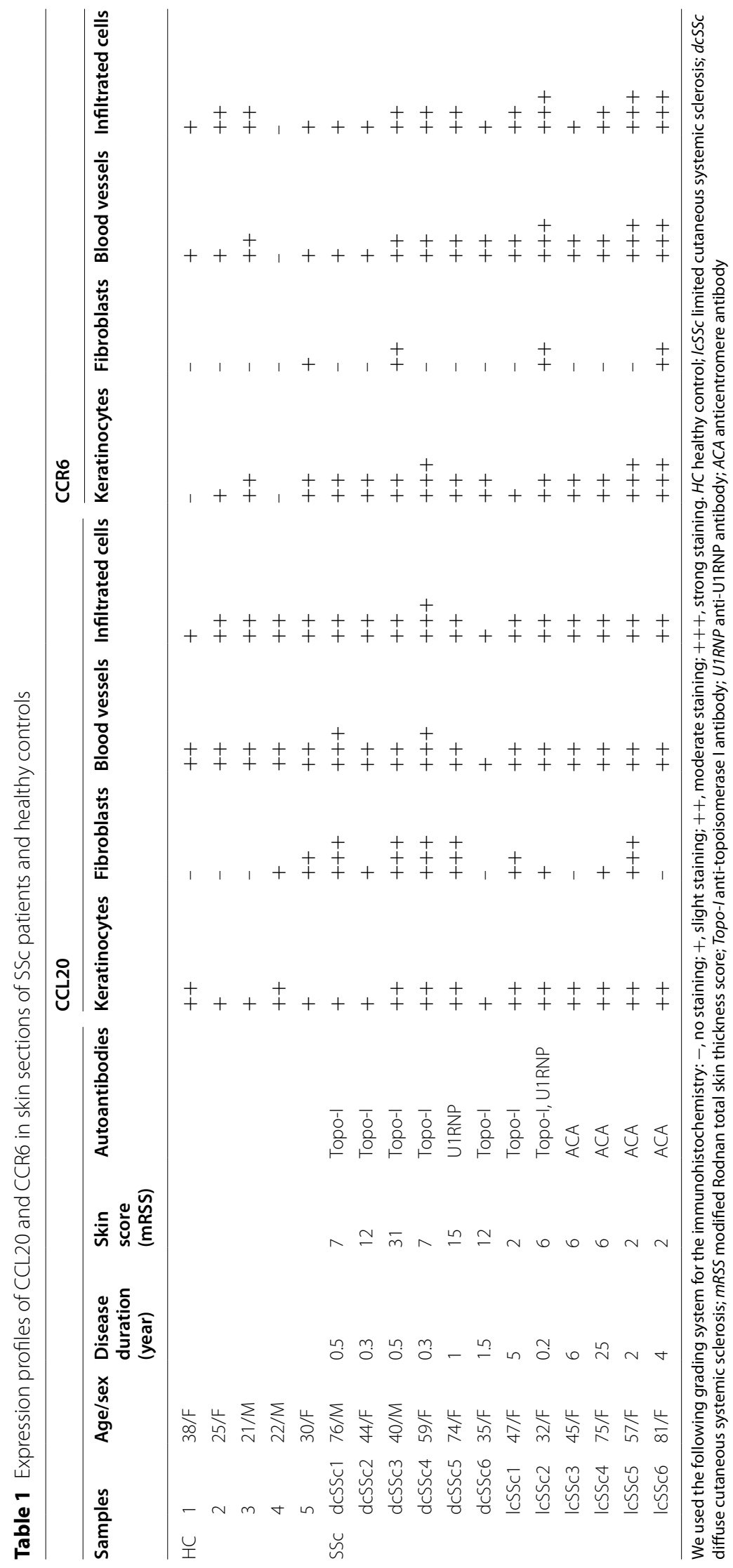


stimulated with recombinant human IL-17A, IL-10, and TGF- $\beta 1$ (all from Peprotech, Rocky Hill, NJ, USA). Cells were then collected using TRIzol Reagent (Thermo Fisher Scientific) for RNA isolation.

\section{RNA isolation and quantitative reverse transcription (qRT)-PCR}

RNA isolation from HDMECs and qRT-PCR were conducted as described previously [30]. The sequences of primers were as follows: FLI1-forward 5'-GGATGGCAA GGAACTGTGTAA-3'， FLI1-reverse 5'-GGTTGTATA GGCCAGCAG-3', CCL20-forward 5'- TTGTGCGTC TCCTCAGTAAAAA-3', CCL20-reverse 5'- GCAAGT GAAACCTCCAACCC-3'; CCR6-forward 5'- GGGGGC TGTCAGTCATCATC-3', CCR6-reverse 5'- CGTAGA GCACAGGGTTCAGG-3'; GAPDH-forward $5^{\prime}$-ACC CACTCCTCCACCTTTGA-3', GAPDH-reverse 5'-CAT ACCAGGAAATGAGCTTGACAA- $3^{\prime}$.

\section{Immunoblotting}

Whole cell lysates of HDMECs treated with FLII siRNA or SCR were subjected to sodium dodecyl sulfate-PAGE (Thermo Fisher Scientific) and immunoblotting. Antibodies used were against $\beta$-actin (Santa Cruz Biotechnology), CCR6, and FLI1 (both from Abcam), followed by horseradish peroxidase (HRP)-conjugated secondary antibody (Cell Signaling Technology). The protein bands were visualized with chemiluminescence (Nacalai Tesque, Kyoto, Japan). The density of each band was quantified with ImageJ software (National Institutes of Health).

\section{Chromatin immunoprecipitation (ChIP) assay}

ChIP assay was conducted using EpiQuik ChIP kit (Epigentek, Farmingdale, NY, USA) as described previously [30]. Putative FLI1 binding site in the CCR6 promoter was predicted using a web site, JASPAR. The primers that amplify fragments of the CCR6 transcript variant 1 ( -252 bp to $-39 \mathrm{bp})$ and transcript variant 2 ( $-1395 \mathrm{bp}$ to $-1186 \mathrm{bp}$ ) were as follows: CCR6 transcript variant $1 /$ Forward, 5'- ACTGCCGTATCCCTTGTGC-3'; CCR6 transcript variant $1 /$ Reverse, $5^{\prime}$ - TGGGAGAATGGA CATTGTGACC-3', CCR6 transcript variant 2/Forward, 5'- TTCTTTCCAGGCAGGCATTG- ${ }^{\prime}$; CCR6 transcript variant $2 /$ Reverse, $5^{\prime}$ - TCCTCCTCATTTCTACCA TCGC- ${ }^{\prime}$. The amplified DNA products were resolved by agarose gel electrophoresis.

\section{In vivo local gene silencing of Ccr6 with atelocollagen} Ccr6 siRNA was transfected to murine skin in vivo using atelocollagen (AteloGene ${ }^{\circledR}$ Local Use "Quick Gelation," Koken, Tokyo, Japan). Ten micromolar of Silencer select Ccr6 siRNA or SCR (both from Thermo Fisher Scientific) was mixed with atelocollagen, $150 \mu \mathrm{L}$ of which was subcutaneously given to shaved lower back of wild-type (WT) mice once a week. After the injection of siRNA, $200 \mathrm{mg}$ of BLM was subcutaneously injected to the same place every day for a week or 4 weeks.

\section{In vivo vascular permeability assay}

Evans blue dye $(0.5 \%)$ (Sigma-Aldrich, St. Louis, MO, USA) in $200 \mu \mathrm{l}$ of $0.9 \%$ saline was injected into the tail vein, and mice were sacrificed in half an hour. The presence of vascular leakage was macroscopically evaluated in the skin.

\section{In vitro transwell permeability assay}

Twenty-four well dishes with inserts (collagen-coated, $0.45-\mathrm{mm}$-meshed) were used for the assay. HDMECs $\left(3 \times 10^{5}\right.$ in $300 \mathrm{~mL}$ of endothelial growth medium) were seeded into each insert. Cells were cultured at $37^{\circ} \mathrm{C}$ for $48 \mathrm{~h}$ to make a monolayer of the cells at the bottom. Then, CCR6 siRNA (final concentration, $10 \mathrm{nM}$ ) was added to the top chamber and cultured for another $48 \mathrm{~h}$. Next, cells were stimulated with $100 \mathrm{ng} / \mathrm{mL}$ of recombinant human tumor necrosis factor- $\alpha$ ( $R \& D$ systems, Minneapolis, MN, USA) for $24 \mathrm{~h}$. After the stimulation, the bottom chambers were refilled with $1 \mathrm{~mL}$ of serum-free media, while serum-free media including $15 \mathrm{ml} / \mathrm{mL}$ of Streptavidin-HRP (R\&D systems) were added in the top chambers. After $30-\mathrm{min}$ incubation at $37^{\circ} \mathrm{C}, 20 \mathrm{~mL}$ of media from the bottom chambers were transferred to a new 96 -well plate in triplicate. Then, $50 \mathrm{~mL}$ of TMB substrate (Abcam) was added in each well and incubated for $5 \mathrm{~min}$ at room temperature. The reaction was stopped by adding $25 \mathrm{ml}$ of stop solution (Abcam) into each well, and absorption at $450 \mathrm{~nm}$ was measured.

\section{In vitro angiogenesis assay}

HDMECs were treated with $20 \mathrm{nM}$ of CCR6 siRNA or SCR (both from Thermo Fisher Scientific) for $48 \mathrm{~h}$. Then, cells were treated with $10 \mu \mathrm{g} / \mathrm{mL}$ of mitomycin C (Sigma Aldrich) for $2 \mathrm{~h}$. A 24-well plate was coated with $250 \mu \mathrm{L}$ of growth factor-reduced Matrigel (BD Biosciences, San Jose, CA, USA). After the gel was solidified, cells were trypsinized and seeded onto the Matrigel at $7 \times 10^{4}$ cells per well and incubated for $24 \mathrm{~h}$. Cells were treated with calcein AM before observation. Five photographs were taken randomly from each well. The numbers of meshes, tubes, and intersections were counted manually.

\section{Statistical analysis}

Statistical analysis was conducted with Welch's $t$ test to compare two unpaired data. Statistical significance was defined as a $P$ value of $<0.05$. 


\section{Results}

The expression profiles of CCL20 and CCR6 in the involved skin of SSc patients

As an initial experiment, we evaluated the expression of CCL20 and CCR6 in skin biopsy samples of SSc patients and healthy controls (Fig. 1 and Table 1). In healthy control skin CCL20 was abundantly expressed in keratinocytes, dermal small vessels, and inflammatory cells, while marginally detected in dermal fibroblasts, as previously reported [31]. In the skin of SSc patients, similar expression profiles to those of healthy controls were observed in keratinocytes, dermal small vessels and inflammatory cells, but an increased trend of CCL20 expression was evident in dermal fibroblasts relative to those cells of healthy control skin (the evaluation with grading scale; median [25-75 percentiles], $1.5[0.25-3.0]$ versus $0[0-1.5], p=0.078)$. Importantly, CCL20 was significantly up-regulated in dermal fibroblasts of dcSSc patients (disease duration of $<2$ years) compared with those cells of healthy controls (3 [0.753 ] versus 0 [0-1.5], $p=0.046)$, which is consistent with a previous finding that CCL20 expression is increased in dermal fibroblasts of early SSc patients [13]. With respect to CCR6, there were detectable signals in various cell types of healthy control skin, but dermal fibroblasts displayed a low expression level relative to the other cell types. In the skin of SSc patients, CCR6 expression was significantly increased in dermal small vessels and keratinocytes as compared to those cells of healthy control skin $(2[2-2.8]$ versus 1 [0.5-1.5], $p=0.022 ; 2.0[2.0-3.0]$ versus $1.0[1.0-2.0], p=0.0089$; respectively), while comparable in dermal fibroblasts and inflammatory cells. We also explored the association between the signal intensity of CCL20/CCR6 in each cell type and modified Rodnan total skin thickness score, but there were no significant correlations (data not shown). Taken together, these results suggest the potential contribution of dermal fibroblasts of early SSc patients to recruiting Th17 cells to the affected skin lesion, as previously reported [13], and the activation of CCL20/CCR6 axis in endothelial cells and keratinocytes of SSc-involved skin. The role of the CCL20/CCR6 axis has been well studied in endothelial cells, while the CCL20/CCR6 axis does not affect the migration and proliferation of keratinocytes [32]; therefore, we focused on endothelial cells in the following experiments.

\section{FLI1 deficiency induces CCR6 expression in dermal microvascular endothelial cells}

Given that Fli1 deficiency reproduces SSc-like properties, including the expression profiles of chemokines, in endothelial cells [26, 27], we examined the effect of FLI1 siRNA on the expression of CCL20 and CCR6 in HDMECs. As shown in Fig. 2A, FLI1 siRNA enhanced CCR6 expression on the mRNA level, while not affecting CCL20 expression. Increased expression of CCR6 by FLI1 depletion was also confirmed on the protein level by immunoblotting (Fig. 2B). Furthermore, chromatin immunoprecipitation revealed the binding of FLI1 to the

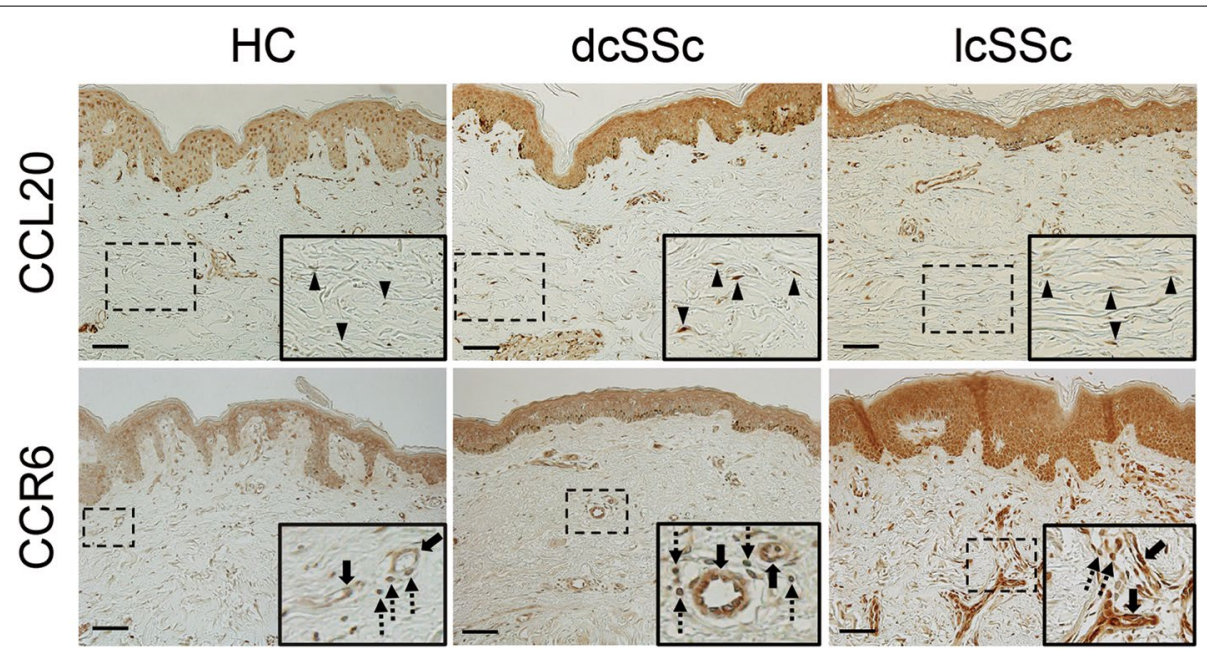

Fig. 1 The expression profiles of CCL20 and CCR6 in SSc patients and healthy controls. The expression of CCL20 and CCR6 was evaluated by immunohistochemistry in the skin sections from diffuse cutaneous SSc (dcSSc) patients, limited cutaneous SSc (IcSSc) patients, and healthy controls (HC). Representative results are shown. Lower panels of each picture exhibit dermal fibroblasts (pointed out with arrowheads) in CCL20 staining and dermal small vessels and inflammatory cells (pointed out with unbroken arrows and dashed arrows, respectively) in CCR6 staining, which are shown with dashed solid squares in upper panels. Horseradish peroxidase activity was detected by 3, 3'-diaminobenzidine. Counterstaining was carried out with methyl green. A scale bar is $100 \mu \mathrm{m}$ 

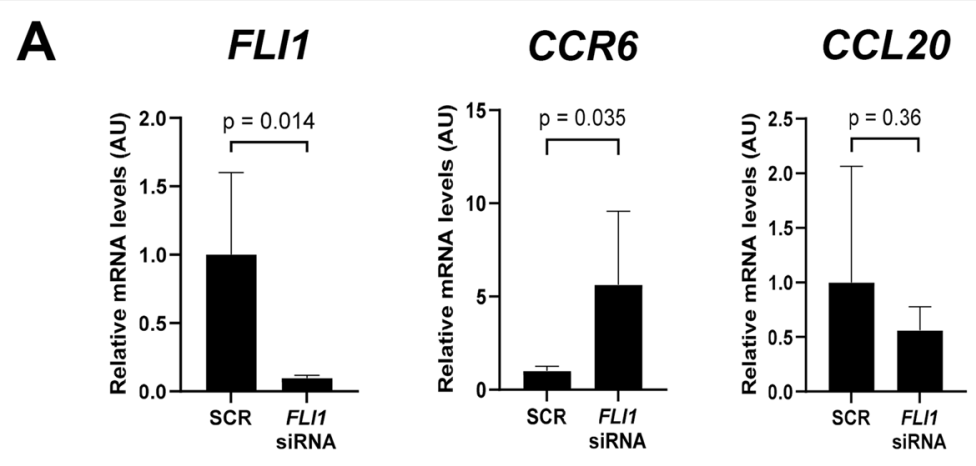

B

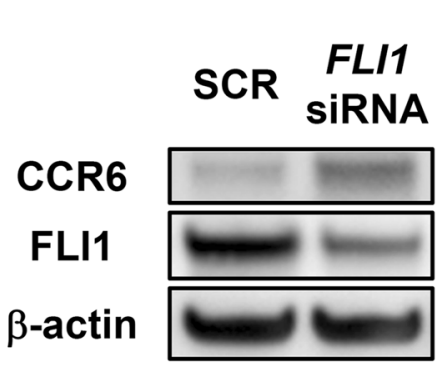

\section{FLI1}

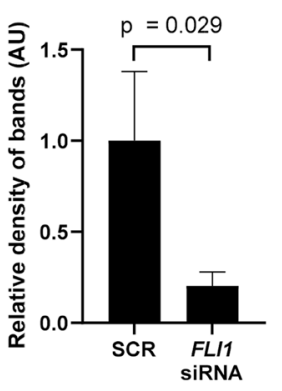

CCR6

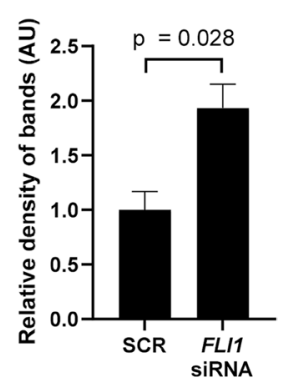

C

HDMECs

HDMECs

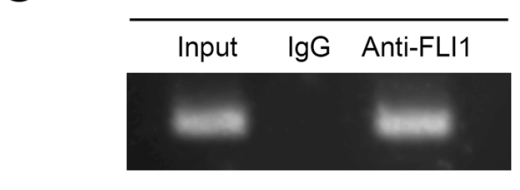

CCR6 transcript variant 1 promoter

-252 to -39

D

\section{CCR6}

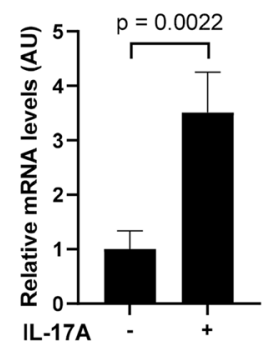

CCR6

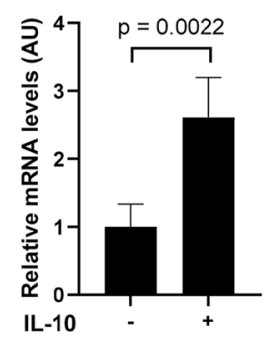

\section{CCR6}

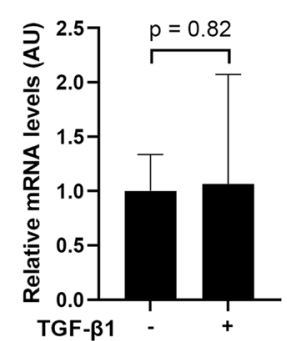

Fig. 2 The contribution of Fli1 deficiency to the up-regulated expression of CCR6 in endothelial cells. A mRNA levels of FLI1, CCL20, and CCR6 in human dermal microvascular endothelial cells (HDMECs) transfected with FLI1 siRNA or non-silencing scrambled RNA (SCR) were examined by qRT-PCR ( $n=6$ for each group). Results are expressed as means \pm SEM. AU, arbitrary unit. B Protein levels of CCR6 and FLI1 in HDMECs transfected with FL/1 siRNA or SCR were examined by immunoblotting ( $n=4$ for each group). C Chromatin was isolated from HDMECs and immunoprecipitated using rabbit anti-FLI1 antibody or rabbit lgG. After isolation of bound DNA, PCR amplification was carried out using two sets of primers specific for the promotes of CCR6 transcript variant 1 (NM_004367) and CCR6 transcript variant 2 (NM_031409). D mRNA levels of CCR6 were determined by qRT-PCR in HDMECs treated with IL-17A (20 ng/mL), IL-10 (20 ng/mL), or TGF- $\beta 1(2 \mathrm{ng} / \mathrm{mL})$

CCR6 promoters of 2 transcript variants (Transcript variant 1; NM_004367, Transcript variant 2; NM_031409) in HDMECs (Fig. 2C). These results suggest that FLI1 serves as a transcriptional repressor of the CCR6 gene and that FLI1 deficiency at least partially contributes to CCR6 upregulation in endothelial cells. 
Given that the CCL20/CCR6 axis plays a critical part in the recruitment of Th17 cells and regulatory $\mathrm{T}$ cells, we also evaluated the effects of cytokines produced by those cells, such as IL-17A, IL-10, and TGF- $\beta 1$, on endothelial CCR6 expression. As shown in Fig. 2D, IL-17A and IL-10, but not TGF- $\beta 1$, enhanced the expression of CCR6 in HDMECs. These results suggest that the recruitment of Th17 cells and regulatory $\mathrm{T}$ cells potentially promotes the expression of CCR6 in endothelial cells, possibly regulating the CCL20/CCR6-dependent angiogenic process related to the pathological inflammation. Considering that IL-17A is up-regulated in the skin of SSc patients $[33,34]$, IL-17A likely contributes to CCR6 induction in dermal microvascular endothelial cells of SSc patients, as well as FLI1 deficiency.

\section{Ccr6 siRNA restores vascular hyperpermeability induced by BLM injection in mice}

To further confirm if CCR6 upregulation is involved in the development of SSc vasculopathy, we evaluated the effect of Ccr6 siRNA on the vascular aspect of BLMtreated mice, a widely recognized animal model of SSc. As a part of SSc-like vascular features induced by BLM injection, we focused on vascular permeability which can be evaluated by Evans blue dye injection [35]. In mice treated with SCR, a 1-week BLM challenge increased the permeability of vasculature in the injected skin area relative to adjacent non-injected areas (a left panel of Fig. 3A). On the other hand, Evans blue dye extravasation was remarkably attenuated after 1-week BLM injection in mice treated with Ccr6 siRNA (a right panel of Fig. 3A). These findings were reproduced by in vitro transwell permeability assay, showing that significantly less streptavidin-HRP permeated through the monolayer of HDMECs treated with CCR6 siRNA as compared to the control monolayer treated with SCR. (Fig. 3B). These results indicate that CCR6 is required for vascular destabilization induced by BLM-dependent inflammation.

To further confirm this finding, we employed immunostaining for $\alpha$-SMA, NG2, and VE-cadherin, markers of vascular stabilization. We used the skin sections of BLM-challenged mice treated with Ccr6 siRNA or SCR for 4 weeks, in which we confirmed CCR6 suppression at the protein level (Fig. $3 C$ ). Generally, $\alpha$-SMA is highly expressed in pericytes engaged in vascular stabilization, while being marginally found in those cells promoting angiogenesis [36, 37]. As shown in Fig. 3D, the expression of $\alpha$-SMA was increased in dermal small vessels (capillaries and venules) of Ccr6 siRNA-treated mice as compared to SCR-treated ones. Another pericytes marker, NG2 proteoglycan, which enhances maturation and formation of endothelial cell junctions by acting as an auxiliary receptor that augments signaling through integrins and growth factor receptors [38], was also stained similarly (Fig. 3E). With respect to VE-cadherin, a key cell adhesion molecule regulating endothelial cell integration and vascular permeability [39], CCR6 siRNA-treated skin exhibited stronger staining in dermal small blood vessels than SCR-treated skin (Fig. 3F). These results indicate that Ccr6 siRNA stabilizes dermal small vessels in BLM-treated mice.

We further sought for the involvement of endothelial apoptosis in vascular permeability of BLM-treated mice because endothelial apoptosis is a part of the vascular process associated with SSc [40]. Immunostaining of cleaved caspase 3 revealed that the positive signals were totally absent in cells constituting dermal blood vessels after BLM injection under either Ccr6 siRNA or SCR treatment, while being evident in some inflammatory cells (Fig. 3G). Thus, the increased vascular permeability was not due to apoptosis of endothelial cells. This finding was consistent with the results of earlier studies demonstrating that apoptosis is mainly observed in inflammatory cells, but not in endothelial cells and dermal fibroblasts, of BLM-treated murine skin [41].

\section{Decreased angiogenic activity of CCR6 siRNA-treated HDMECs}

As described above, CCR6 downregulation was associated with vascular stabilization in BLM-treated mice. Generally, vascular stabilization is associated with reduced angiogenic activity, suggesting that CCR6 reduction suppresses the pro-angiogenic activity of endothelial cells. To address this issue, we employed in vitro angiogenesis assay with the Matrigel. As shown in the top panels of Fig. 4A, SCR-treated HDMECs formed favorable tube networks, whereas CCR6 siRNAtreated HDMECs showed relatively large tube networks under the same culture condition. To objectively evaluate the activity of angiogenesis, we looked at the numbers of meshes, tubes and intersections. Of note, the numbers of meshes (91 [87-92] versus 23 [21-29.5], $p<0.001)$, tubes (180 [160.5-198] versus 45 [38.5-57], $p<0.001)$ and intersections (124 [100.5-130.5] versus $29[20.5-38.5], p<0.001)$ were decreased significantly in CCR6 siRNA-treated HDMECs compared with SCR-treated HDMECs (bottom graphs of Fig. 4A). Taken together, CCR6 downregulation suppresses the pro-angiogenic activity of endothelial cells, which at least partially underlies vascular stabilization in Ccr6 siRNA-treated BLM-injected mice. This notion was also confirmed in vivo by the decreased number of dermal small vessels in Ccr6 siRNA-treated BLM-injected mice as compared to SCR-treated ones (Fig. 4B). 

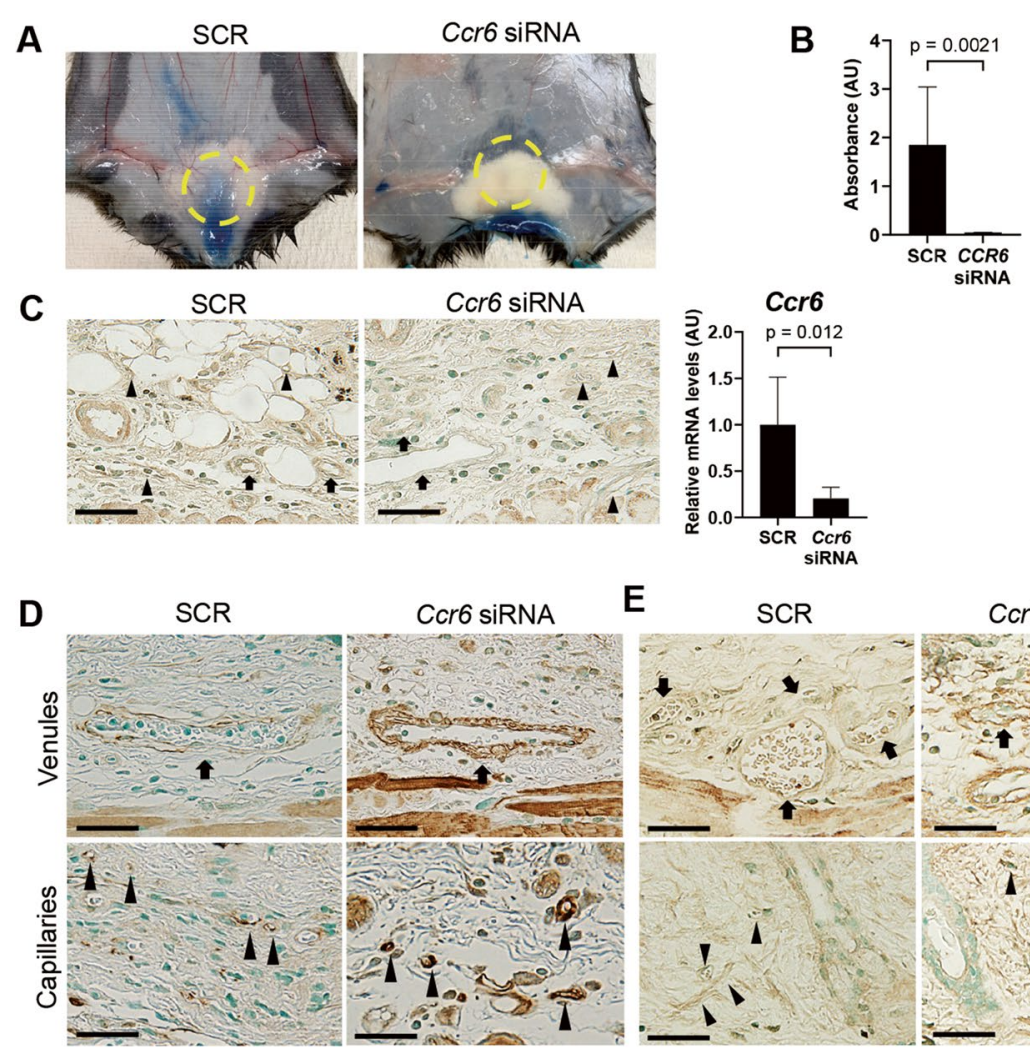

E SCR
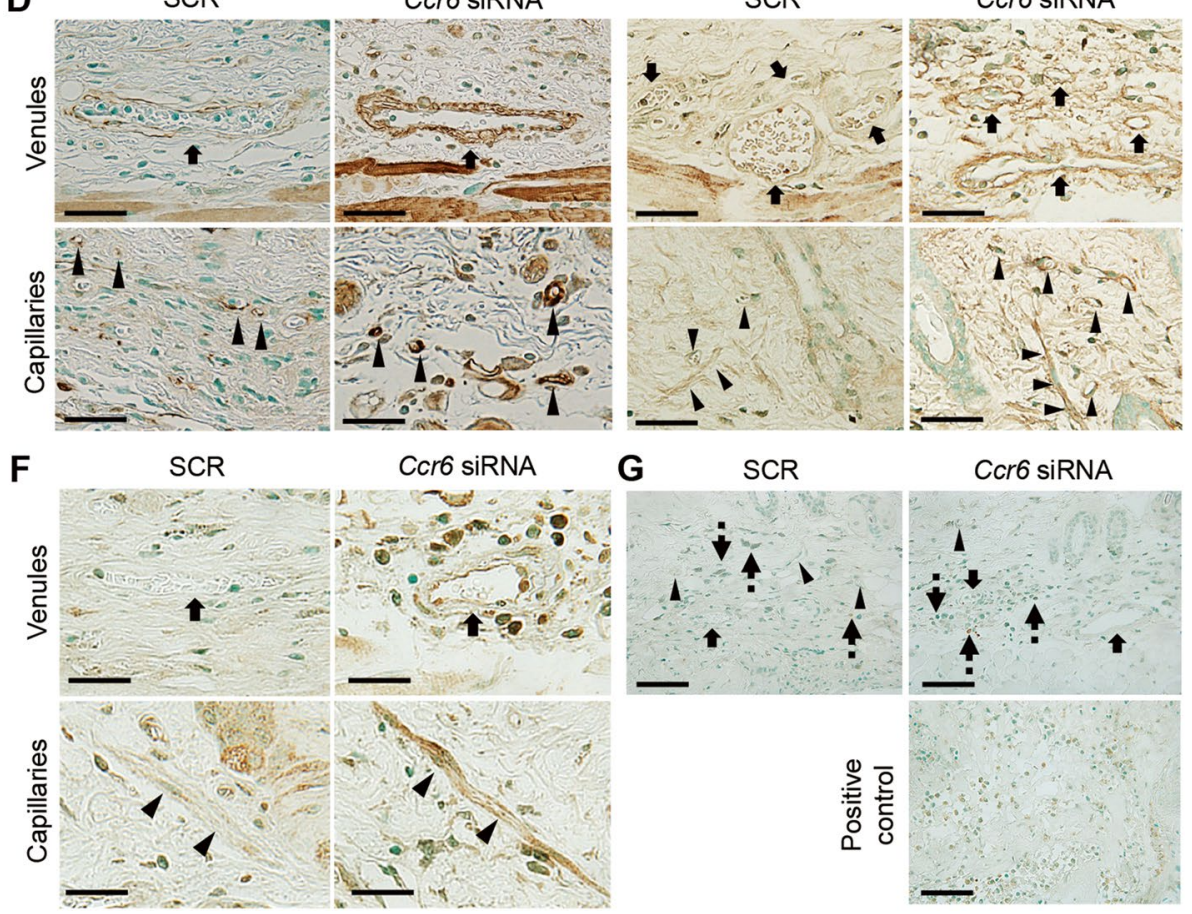

Fig. 3 Ccr6 gene silencing restores BLM-induced vascular hyperpermeability in mice. Wild-type mice were injected with Ccr6 siRNA or scrambled non-silencing RNA (SCR), followed by 1-week and 4-week bleomycin (BLM) challenge. A The leakage of Evans blue dye was macroscopically evaluated in the skin after 1-week BLM injection. $\mathbf{B}$ In vitro transwell permeability assay showed decreased permeability in the monolayer of HDMECs treated with CCR6 siRNA relative to the control cells treated with SCR. C-F After 4-week BLM injection, CCR6 was visualized by immunohistochemistry (left photographs of C). CCR6 knockdown was also confirmed by qRT-PCT with the whole skin sections (a right graph of C). a-smooth muscle actin (D), NG2 (E), and VE-cadherin (F) were visualized by immunohistochemistry. Apoptotic cells were visualized by staining cleaved caspase $3(\mathbf{G})$. Skin sections of angiosarcoma were used as positive controls (a bottom panel of G). Unbroken arrows, arrow heads, and dashed arrows in each figure represent venules, capillaries, and inflammatory cells, respectively. Each graph indicates mean \pm SEM of the indicated parameters. A scale bar is $25 \mu \mathrm{m}$. AU, arbitrary unit. Representative results of 6 independent experiments were shown

\section{Discussion}

This study was undertaken to investigate the role of the CCL20/CCR6 axis in the development of SSc vasculopathy because serum CCL20 levels correlate with mPAP values in SSc patients [6]. Given that mPAP is positively correlated with the severity of nailfold capillary changes
[42], it was postulated that the activation of the CCL20/ CCR6 axis plays a part in the destabilization of dermal small vessels. Based on this idea, we evaluated the role of the CCL20/CCR6 axis in the regulation of endothelial behaviors. Our initial study with immunochemistry demonstrated the up-regulated expression of CCR6 in dermal 

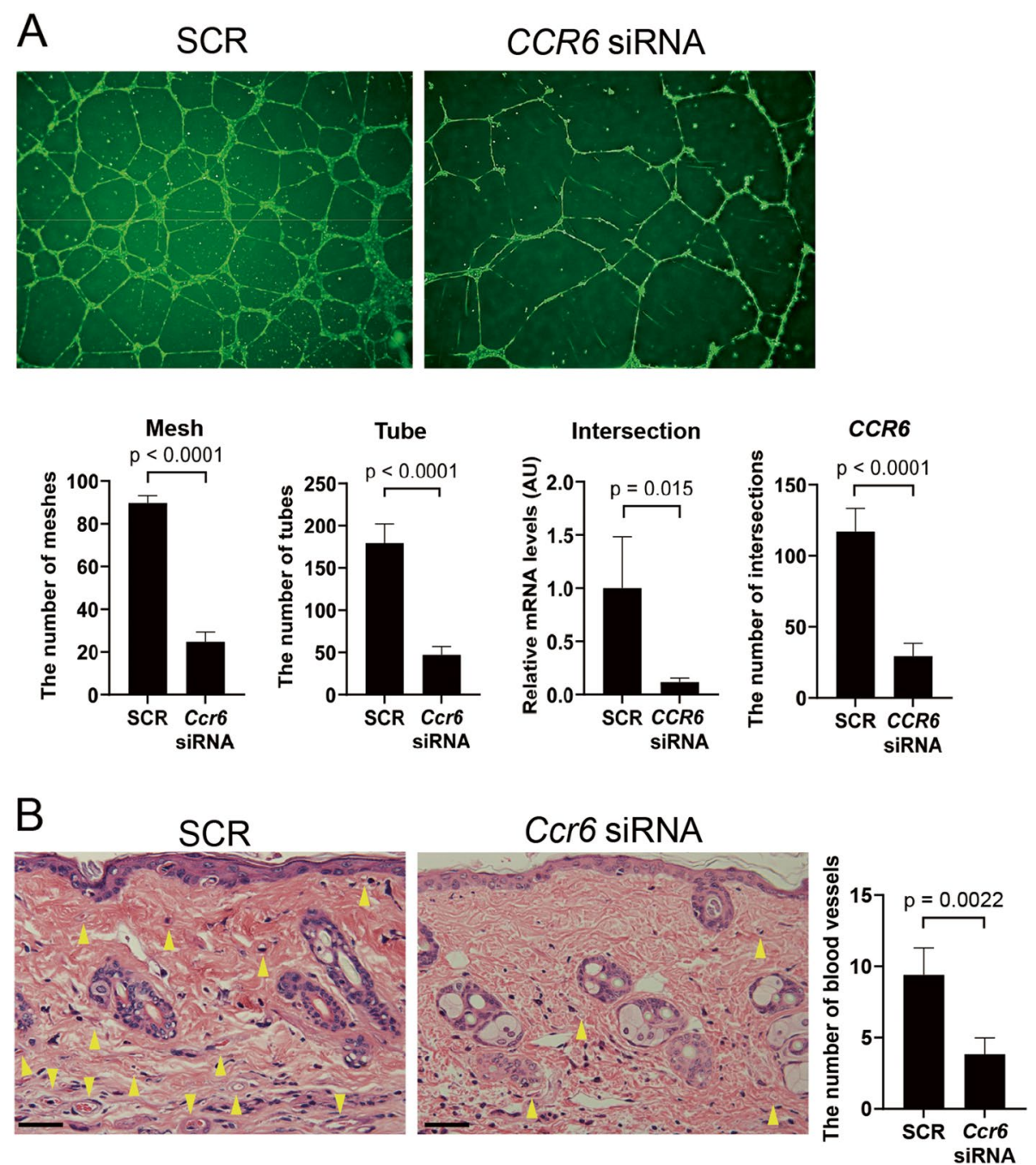

Fig. 4 CCR6 siRNA suppresses tubulogenic activity of HDMECs and in vivo neovascularization. A Tube formation assay was performed by applying HDMECs treated with Ccr6 siRNA or scrambled non-silencing RNA (SCR) onto the Matrigel and incubating for $24 \mathrm{~h}$. To eliminate the influence of proliferation, cells were treated with mitomycin C before the assay. Representative images are shown ( $n=5$ for each group). The numbers of meshes, tubes, and intersections were counted manually. CCR6 mRNA levels were determined by qRT-PCR. B The number of dermal microvessels in the skin of BLM-treated mice subjected to C Cr6 siRNA or SCR was counted manually in the specimen subjected to hematoxylin and eosin staining ( $n=6$ for each group). Each graph represents mean \pm SEM of the indicated parameters

microvascular endothelial cells of SSc patients. Further cell culture studies revealed the upregulation of CCR6 by FLI1 deficiency, a critical predisposing factor of SSc, in HDMECs. More importantly, Ccr6 siRNA restored BLM-induced vascular hyperpermeability in mice. Taken together with evidence that the CCL20/CCR6 axis functions as a potent pro-angiogenic regulator [43], CCR6 upregulation may be involved in the development of SSc vasculopathy.

A previous study by Tao et al. [13] demonstrated the increased expression of CCL20 in dermal fibroblasts of early SSc patients relative to those cells of healthy control skin, suggesting that CCL20 produced by dermal fibroblasts contributes to Th17 infiltration into the involved skin of patients with early SSc. Supporting this previous finding, we found a significant elevation of CCL20 in dermal fibroblasts of dcSSc patients with a disease duration of $<2$ years. Under the physiological condition, CCL20 is abundantly expressed by keratinocytes and endothelial cells, but its expression is relatively low in dermal fibroblasts, as shown in the current and previous studies [31]. Therefore, CCL20 upregulation seems to be a characteristic feature of SSc dermal fibroblasts. With respect to the expression levels of CCR6, our results 
were also consistent with the study by Tao et al. [13] in that the elevation of CCR6 was observed significantly in keratinocytes and slightly in dermal fibroblasts and infiltrated cells. In addition, our current study disclosed a significant elevation of CCR6 in dermal small vessels of SSc patients, especially in lcSSc patients, irrespective of disease duration. Taken together, these results suggest that CCL20 secreted from dermal fibroblasts plays a role in the early stage of dcSSc (disease duration of $<2$ years), while CCR6 up-regulation in endothelial cells contributes to the development of SSc vasculopathy throughout the whole disease course. This notion is likely plausible because SSc-related PAH, which is linked to elevated serum CCL20 levels, is a complication frequently seen in lcSSc patients with a long disease history.

SSc vasculopathy is characterized by vascular structural changes, such as arteriolar stenosis, capillary dilation, and capillary loss [40]. The chronological capillary changes are well documented in nailfold capillaries [44]. The initial changes are capillary dilation and bleeding, reflecting vascular destabilization. These changes are followed by capillary loss, finally leading to abnormal angiogenic changes, such as ramified capillary. These vascular alterations are caused by dysregulated angiogenesis and defective vasculogenesis. FLI1 deficiency is a key disease factor regulating a broad spectrum of endothelial behaviors and vascular remodeling associated with SSc vasculopathy, including angiogenesis and vasculogenesis [45]. FLI1 deficiency suppresses the expression of CD31, VEcadherin, $\mathrm{S}_{1} \mathrm{P}_{1}$ and platelet-derived growth factor-B in endothelial cells, while upregulating matrix metalloproteinase-9, resulting in vascular destabilization and angiogenesis [29]. The expression of CCN1, which regulates the recruitment of circulating endothelial progenitor cells, is decreased in FLI1-deficient endothelial cells [23], at least partly contributing to defective vasculogenesis. In the current study, CCR6 was up-regulated in dermal microvascular endothelial cells of SSc-involved skin, and FLI1 deficiency increased CCR6 expression in HDMECs. Given that FLI1 bound the CCR6 promoter in HDMECs, CCR6 is a member of molecules involved in the mechanism by which FLI1 deficiency promotes the development of SSc vasculopathy.

Chemokines are initially recognized as a family of proteins recruiting inflammatory cells to the specific tissues and organs. On the other hand, various chemokines have been shown to serve as regulators of angiogenesis associated with inflammation. For instance, CXC chemokines with glutamic acid-leucine-arginine (ELR) motif in their $\mathrm{N}$ terminus are potent promoters of angiogenesis, while those without ELR motif are potent inhibitors of angiogenesis [46]. Of note, the various CXC chemokines, such as CXCL4 (ELR-) [47], CXCL5 (ELR+) [26], CXCL6
(ELR+) [27], CXCL12 (ELR-) [48], CXCL13 (ELR-) [24], and CXCL14 (ELR-) [49], are thought to be associated with the development of SSc vasculopathy. With respect to $\mathrm{CC}$ chemokines, the broad-spectrum inhibitor of $\mathrm{CC}$ chemokines, $35 \mathrm{~K}$, suppresses inflammation-driven angiogenesis, whereas preserving physiological ischemiamediated angiogenesis [50]. Thus, the inhibition of chemokines may be an alternative therapeutic strategy against disease-related inflammatory vascular changes without the undesirable effects on physiological angiogenesis. Considering this point, the blockade of CCR6 is likely a potential therapeutic strategy against SSc vasculopathy. Further studies are required to clarify this point in the future.

There are several limitations in this study. The first limitation is the lack of data on the systemic inhibition of CCR6 in BLM-treated mice. This is a critical point because CCR6 is a chemoattractant receptor of Th17 cells and regulatory $\mathrm{T}$ cells. In this study, we used the local injection of atelocollagen mixed with Ccr6 siRNA in the back skin of BLM-treated mice, in which CCR6 expression in circulating $\mathrm{CD} 4^{+} \mathrm{T}$ cells was not altered (data not shown). To elucidate the effect of systemic CCR6 inhibition, we need to investigate whether anti-CCR6 neutralizing antibody affects skin fibrosis or other vascular changes, such as PAH, using BLM-treated mice and other SSc animal models recapitulating SSc-PAH. The second limitation is that we did not assess the association of the CCR6-dependent angiogenic process and CCR6-dependent recruitment of inflammatory cells. Given that IL-17A enhanced CCR6 expression in HDMECs, Th17-skewed immune polarization may regulate the angiogenic process through CCR6 in the context of SSc pathogenesis. The third limitation is that there remains a room for discussion about the differential effects of CCR6 and CCL20 knockdown on SSc-like pathological events. We currently looked at the effect of CCR6 deletion to examine the role of the CCL20/CCR6 axis, but the targeting of CCL20 should be investigated as well in order to clarify which target is more appropriate to restore the disease process associated with CCL20/CCR6 axis in the context of SSc pathology.

\section{Conclusion}

This is the first report demonstrating a potential contribution of CCR6 to the development of SSc vasculopathy as an inflammation-associated angiogenic factor. These findings suggest that the modification of CCR6 expression can be an effective intervention for SSc vascular symptoms. Also, the induction of CCR6 expression in FLI1-deficient endothelial cells further supports the canonical idea that FLI1 deficiency is a critical disease factor of SSc. 


\begin{abstract}
Abbreviations
SSc: Systemic sclerosis; mPAP: Mean pulmonary arterial pressure; Th: Thelper; FLI1: Friend leukemia virus integration 1; a-SMA: a-Smooth muscle actin; VEcadherin: Vascular endothelial-cadherin; dcSSc: Diffuse cutaneous SSc; ICSSc: Limited cutaneous SSc; HDMECs: Human dermal microvascular endothelial cells; SCR: Non-silencing scrambled RNA; qRT-PCR: Quantitative reverse transcription-PCR; ChIP: Chromatin immunoprecipitation; WT: Wild-type; PAH: Pulmonary arterial hypertension; ELR: Glutamic acid-leucine-arginine; HC: Healthy controls; BLM: Bleomycin.
\end{abstract}

\section{Acknowledgements}

Not applicable.

\section{Authors' contributions}

Conception and design, $\mathrm{TI}, \mathrm{YA}$, and SS; acquisition of data, TI, TM, YF, ST, JO, KA, $\mathrm{YN}, \mathrm{YW}$, and $\mathrm{AY}$; analysis and interpretation of data, TI, YA, and SS; obtaining funding; $Y A$ and $S S$; supervision, $Y A, A Y$, and SS. The author(s) read and approved the final manuscript.

\section{Funding}

This work was supported by a grant for Research on Intractable Diseases from the Ministry of Health, Labour, and Welfare of Japan.

\section{Availability of data and materials \\ Not applicable.}

\section{Declarations}

\section{Ethics approval and consent to participate}

The study was conducted according to the guidelines of the Declaration of Helsinki and approved by the ethical committee and the committee on animal experimentation of the University of Tokyo Graduate School of Medicine. Written informed consent was obtained from all participants.

\section{Consent for publication}

Not applicable.

\section{Competing interests}

The authors declare that they have no competing interests.

Received: 20 June 2021 Accepted: 31 October 2021

Published online: 13 November 2021

\section{References}

1. Asano Y. Systemic sclerosis. J Dermatol. 2018;45(2):128-38.

2. Khanna D, Lin CJF, Furst DE, Goldin J, Kim G, Kuwana M, et al. Tocilizumab in systemic sclerosis: a randomised, double-blind, placebo-controlled, phase 3 trial. Lancet Respir Med. 2020;8(10):963-74.

3. Matucci-Cerinic M, Denton CP, Furst DE, Mayes MD, Hsu VM, Carpentier $P$, et al. Bosentan treatment of digital ulcers related to systemic sclerosis: results from the RAPIDS-2 randomised, double-blind, placebo-controlled trial. Ann Rheum Dis. 2011;70(1):32-8.

4. Asano Y, Varga J. Rationally-based therapeutic disease modification in systemic sclerosis: novel strategies. Semin Cell Dev Biol. 2019.

5. King J, Abraham D, Stratton R. Chemokines in systemic sclerosis. Immunol Lett. 2018;195:68-75.

6. Ikawa T, Miyagawa T, Fukui Y, Minatsuki S, Maki H, Inaba T, et al. Association of serum CCL20 levels with pulmonary vascular involvement and primary biliary cholangitis in patients with systemic sclerosis. Int J Rheum Dis. 2021;24(5):711-8.

7. Schutyser E, Struyf S, Van Damme J. The CC chemokine CCL20 and its receptor CCR6. Cytokine Growth Factor Rev. 2003;14(5):409-26.

8. Furue $\mathrm{K}$, Ito T, Tsuji G, Nakahara T, Furue M. The CCL2O and CCR6 axis in psoriasis. Scand J Immunol. 2020;91(3):e12846.
9. Furue K, Ulzii D, Tanaka Y, Ito T, Tsuji G, Kido-Nakahara M, et al. Pathogenic implication of epidermal scratch injury in psoriasis and atopic dermatitis. J Dermatol. 2020;47(9):979-88.

10. Trivedi PJ, Adams DH: Chemokines and chemokine receptors as therapeutic targets in inflammatory bowel disease; Pitfalls and Promise. $J$ Crohns Colitis 2018, 12(suppl_2):S641-s652.

11. Lee AY, Korner H. CC chemokine receptor 6 (CCR6) in the pathogenesis of systemic lupus erythematosus. Immunol Cell Biol. 2020;98(10):845-53.

12. Page G, Chevrel G, Miossec P. Anatomic localization of immature and mature dendritic cell subsets in dermatomyositis and polymyositis: interaction with chemokines and Th1 cytokine-producing cells. Arthritis Rheum. 2004;50(1):199-208.

13. Tao J, Li L, Tan Z, Li Y, Yang J, Tian F, et al. Up-regulation of CC chemokine ligand 20 and its receptor CCR6 in the lesional skin of early systemic sclerosis. Eur J Dermatol. 2011;21(5):731-6.

14. Hippe A, Braun SA, Oláh P, Gerber PA, Schorr A, Seeliger S, et al. EGFR/Rasinduced CCL20 production modulates the tumour microenvironment. $\mathrm{Br}$ J Cancer. 2020;123(6):942-54.

15. Kubo M, Czuwara-Ladykowska J, Moussa O, Markiewicz M, Smith E, Silver RM, et al. Persistent down-regulation of Fli1, a suppressor of collagen transcription, in fibrotic scleroderma skin. Am J Pathol. 2003;163(2):571-81.

16. Takahashi T, Asano Y, Sugawara K, Yamashita T, Nakamura K, Saigusa R, et al. Epithelial Fli1 deficiency drives systemic autoimmunity and fibrosis: possible roles in scleroderma. J Exp Med. 2017;214(4):1129-51.

17. Yamashita K, Kawasaki A, Matsushita T, Furukawa H, Kondo Y, Okiyama N, et al. Association of functional (GA) $n$ microsatellite polymorphism in the FLI1 gene with susceptibility to human systemic sclerosis. Rheumatology (Oxford). 2020;59(11):3553-62

18. Asano Y. Epigenetic suppression of Fli1, a potential predisposing factor in the pathogenesis of systemic sclerosis. Int J Biochem Cell Biol. 2015;67:86-91.

19. Taniguchi $T$, Asano $Y$, Akamata $K$, Noda S, Takahashi T, Ichimura Y, et al, Fibrosis, vascular activation, and immune abnormalities resembling systemic sclerosis in bleomycin-treated Fli-1-haploinsufficient mice. Arthritis Rheumatol. 2015;67(2):517-26.

20. Ichimura Y, Asano Y, Akamata K, Noda S, Taniguchi T, Takahashi T, et al. Progranulin overproduction due to Fli-1 deficiency contributes to the resistance of dermal fibroblasts to tumor necrosis factor in systemic sclerosis. Arthritis Rheumatol. 2015;67(12):3245-55.

21. Saigusa R, Asano Y, Nakamura K, Hirabayashi M, Miura S, Yamashita T, et al. Systemic sclerosis dermal fibroblasts suppress Th1 cytokine production via galectin-9 overproduction due to Fli1 deficiency. J Invest Dermatol. 2017.

22. Saigusa R, Asano Y, Yamashita T, Taniguchi T, Takahashi T, Ichimura Y, et al. Fli1 deficiency contributes to the downregulation of endothelial protein C receptor in systemic sclerosis: a possible role in prothrombotic conditions. Br J Dermatol. 2016;174(2):338-47.

23. Saigusa R, Asano Y, Taniguchi T, Yamashita T, Takahashi T, Ichimura Y, et al. A possible contribution of endothelial CCN1 downregulation due to Fli1 deficiency to the development of digital ulcers in systemic sclerosis. Exp Dermatol. 2015;24(2):127-32.

24. Taniguchi T, Miyagawa T, Toyama S, Yamashita T, Nakamura K, Saigusa R, et al. CXCL13 produced by macrophages due to Fli1 deficiency may contribute to the development of tissue fibrosis, vasculopathy and immune activation in systemic sclerosis. Exp Dermatol. 2018;27(9):1030-7.

25. Miura S, Watanabe Y, Saigusa R, Yamashita T, Nakamura K, Hirabayashi M, et al. Fli1 deficiency suppresses RALDH1 activity of dermal dendritic cells and related induction of regulatory $T$ cells: a possible role in scleroderma. Arthritis Res Ther. 2021;23(1):137.

26. Ichimura Y, Asano Y, Akamata K, Takahashi T, Noda S, Taniguchi T, et al. Fli1 deficiency contributes to the suppression of endothelial CXCL5 expression in systemic sclerosis. Arch Dermatol Res. 2014;306(4):331-8.

27. Taniguchi T, Asano Y, Nakamura K, Yamashita T, Saigusa R, Ichimura Y, et al. Fli1 deficiency induces CXCL6 expression in dermal fibroblasts and endothelial cells, contributing to the development of fibrosis and vasculopathy in systemic sclerosis. J Rheumatol. 2017;44(8):1198-205.

28. Miyagawa T, Taniguchi T, Saigusa R, Fukayama M, Takahashi T, Yamashita $\mathrm{T}$, et al. Fli1 deficiency induces endothelial adipsin expression, contributing to the onset of pulmonary arterial hypertension in systemic sclerosis. Rheumatology (Oxford). 2020;59(8):2005-15. 
29. Asano Y, Stawski L, Hant F, Highland K, Silver R, Szalai G, et al. Endothelial Fli1 deficiency impairs vascular homeostasis: a role in scleroderma vasculopathy. Am J Pathol. 2010;176(4):1983-98.

30. Ichimura Y, Asano Y, Akamata K, Takahashi T, Noda S, Taniguchi T, et al. Fli1 deficiency contributes to the suppression of endothelial CXCL5 expression in systemic sclerosis. Arch Dermatol Res. 2013.

31. Charbonnier AS, Kohrgruber N, Kriehuber E, Stingl G, Rot A, Maurer D. Macrophage inflammatory protein 3alpha is involved in the constitutive trafficking of epidermal langerhans cells. J Exp Med. 1999;190(12):1755-68

32. Kroeze KL, Boink MA, Sampat-Sardjoepersad SC, Waaijman T, Scheper RJ, Gibbs S. Autocrine regulation of re-epithelialization after wounding by chemokine receptors CCR1, CCR10, CXCR1, CXCR2, and CXCR3. J Invest Dermatol. 2012;132(1):216-25.

33. Nakashima T, Jinnin M, Yamane K, Honda N, Kajihara I, Makino T, et al. Impaired IL-17 signaling pathway contributes to the increased collagen expression in scleroderma fibroblasts. J Immunol. 2012;188(8):3573-83.

34. Truchetet ME, Brembilla NC, Montanari E, Lonati P, Raschi E, Zeni S, et al. Interleukin-17A+ cell counts are increased in systemic sclerosis skin and their number is inversely correlated with the extent of skin involvement. Arthritis Rheum. 2013;65(5):1347-56

35. Saigusa R, Asano Y, Taniguchi T, Yamashita T, Ichimura Y, Takahashi T, et al. Multifaceted contribution of the TLR4-activated IRF5 transcription factor in systemic sclerosis. Proc Natl Acad Sci U S A. 2015;112(49):15136-41.

36. von Tell D, Armulik A, Betsholtz C. Pericytes and vascular stability. Exp Cell Res. 2006;312(5):623-9.

37. Alarcon-Martinez $L$, Yemisci M, Dalkara T. Pericyte morphology and function. Histol Histopathol. 2021;18314.

38. Stallcup WB. The NG2 proteoglycan in pericyte biology. Adv Exp Med Biol. 2018;1109:5-19

39. Vestweber D. VE-cadherin: the major endothelial adhesion molecule controlling cellular junctions and blood vessel formation. Arterioscler Thromb Vasc Biol. 2008;28(2):223-32.

40. Asano Y, Sato S. Vasculopathy in scleroderma. Semin Immunopathol. 2015;37(5):489-500

41. Yamamoto T, Nishioka K. Possible role of apoptosis in the pathogenesis of bleomycin-induced scleroderma. J Invest Dermatol. 2004;122(1):44-50.
42. Riccieri V, Vasile M, lannace N, Stefanantoni K, Sciarra I, Vizza CD, et al. Systemic sclerosis patients with and without pulmonary arterial hypertension: a nailfold capillaroscopy study. Rheumatology (Oxford). 2013;52(8):1525-8.

43. Benkheil M, Van Haele M, Roskams T, Laporte M, Noppen S, Abbasi K, et al. CCL20, a direct-acting pro-angiogenic chemokine induced by hepatitis $\mathrm{C}$ virus (HCV): potential role in HCV-related liver cancer. Exp Cell Res. 2018;372(2):168-77

44. Cutolo M, Sulli A, Secchi ME, Paolino S, Pizzorni C: Nailfold capillaroscopy is useful for the diagnosis and follow-up of autoimmune rheumatic diseases. A future tool for the analysis of microvascular heart involvement? Rheumatology (Oxford) 2006, 45 Suppl 4:iv43-46.

45. Nakamura K, Taniguchi T, Hirabayashi M, Yamashita T, Saigusa R, Miura $\mathrm{S}$, et al. Altered properties of endothelial cells and mesenchymal stem cells underlying the development of scleroderma-like vasculopathy in KLF5(+/-) ;Fli-1(+/-) Mice. Arthritis Rheumatol. 2020;72(12):2136-46.

46. Belperio JA, Keane MP, Arenberg DA, Addison CL, Ehlert JE, Burdick MD, et al. CXC chemokines in angiogenesis. J Leukoc Biol. 2000;68(1):1-8.

47. van Bon L, Affandi AJ, Broen J, Christmann RB, Marijnissen RJ, Stawski $\mathrm{L}$, et al. Proteome-wide analysis and CXCL4 as a biomarker in systemic sclerosis. N Engl J Med. 2014;370(5):433-43.

48. Ikawa T, Miyagawa T, Fukui Y, Toyama S, Omatsu J, Awaji K, et al. Association of serum CXCL12 levels with arthropathy in patients with systemic sclerosis. Int J Rheum Dis. 2021;24(2):260-7.

49. Fukui Y, Miyagawa T, Hirabayashi M, Yamashita T, Saigusa R, Miura S, et al. Possible association of decreased serum CXCL14 levels with digital ulcers in patients with systemic sclerosis. J Dermatol. 2019;46(7):584-9.

50. Ridiandries A, Tan JT, Ravindran D, Williams H, Medbury HJ, Lindsay L, et al. CC-chemokine class inhibition attenuates pathological angiogenesis while preserving physiological angiogenesis. FASEB J. 2017;31(3):1179-92.

\section{Publisher's Note}

Springer Nature remains neutral with regard to jurisdictional claims in published maps and institutional affiliations.
Ready to submit your research? Choose BMC and benefit from:

- fast, convenient online submission

- thorough peer review by experienced researchers in your field

- rapid publication on acceptance

- support for research data, including large and complex data types

- gold Open Access which fosters wider collaboration and increased citations

- maximum visibility for your research: over $100 \mathrm{M}$ website views per year

At BMC, research is always in progress.

Learn more biomedcentral.com/submissions 